\title{
Time Calibration of the J-PET Detector
}

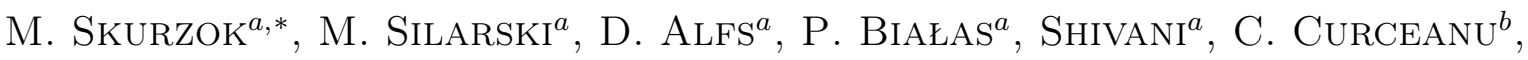

E. Czerwiński ${ }^{a}$, K. Dulski ${ }^{a}$, A. Gajos ${ }^{a}$, B. GŁowacz ${ }^{a}$, M. Gorgol $^{a}$, B.C. Hiesmayr ${ }^{c}$,

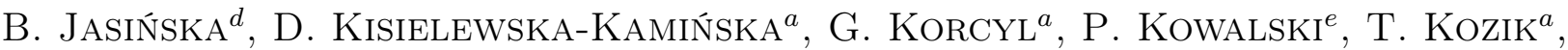
N. KrawCZYK ${ }^{a}$, W. Krzemień ${ }^{f}$, E. Kubicz $^{a}$, Muhsin Mohammed $^{a, g}$, M. PAWlik-NiedźWiecka ${ }^{a}$, S. NiedźWIECKi ${ }^{a}$, M. PAŁKA $^{a}$, L. RACZYŃSKi $^{e}$, J. RAJ $^{a}$, Z. Rudy ${ }^{a}$, N.G. Sharma ${ }^{a}$, S. Sharma ${ }^{a}$, R.Y. Shopa ${ }^{e}$, A. WieczoreK ${ }^{a}$, W. Wiślicki $^{f}$, B. ZGARDZińsKA ${ }^{d}$, M. ZiELIŃSKI ${ }^{a}$ AND P. MOSKAL ${ }^{a}$

${ }^{a}$ Institute of Physics, Jagiellonian University, S. Łojasiewicza 11, 30-348 Kraków, Poland

${ }^{b}$ INFN, Laboratori Nazionali di Frascati, 00044 Frascati, Italy

${ }^{c}$ Faculty of Physics, University of Vienna, 1090 Vienna, Austria

${ }^{d}$ Institute of Physics, M. Curie-Skłodowska University, 20-031 Lublin, Poland

${ }^{e}$ Laboratory for Information Technologies, National Centre for Nuclear Research, 05-400 Otwock-Świerk, Poland ${ }^{f}$ High Energy Physics Division, National Centre for Nuclear Research, 05-400 Otwock-Świerk, Poland

${ }^{g}$ Department of Physics, College of Education for Pure Sciences, University of Mosul, Mosul, Iraq

The Jagiellonian positron emission tomograph project carried out in the Institute of Physics of the Jagiellonian University is focused on construction and tests of the first prototype of PET scanner for medical diagnostic which allows for the simultaneous 3D imaging of the whole human body using organic scintillators. The J-PET prototype consists of 192 scintillator strips forming three cylindrical layers which are optimized for the detection of photons from the electron-positron annihilation with high time-and high angular resolutions. In this article we present time calibration and synchronization of the whole J-PET detection system by irradiating each single detection module with a ${ }^{22} \mathrm{Na}$ source and a small detector providing common reference time for synchronization of all the modules.

DOI: 10.12693/APhysPolA.132.1641

PACS/topics: 06.20.fb, 36.10.Dr, 11.30.Er, 24.80.+y

\section{Introduction}

Positron emission tomography (PET) imaging is a very important tool in medical diagnostics, in particular in oncology, cardiology, neurology, gastrology, and psychiatry. Currently, all commercial PET devices are built with scintillation crystals [1-3]. There are few known methods for PET scanners using time calibration. Timeof-flight (TOF)-PET synchronisation is carried out with radioactive isotopes like sodium or germanium, placed inside the PET device, typically in its geometric center. The gamma quanta from radioactive source are scattered (due to applied shield) allowing synchronisation of all PET components $[4,5]$. There are also methods for time synchronization using several radioactive sources simultaneously [6] as well as using a rotating source along the scintillation chamber [7].

The Jagiellonian PET (J-PET) is the first positron emission tomography scanner built from plastic scintillators which, as organic detectors, are relatively cheap and easy to shape as well as are characterized by very good time measurement resolution. In the J-PET detection system, information about the place of $\gamma$ quanta interaction is extracted solely from timing measurement

*corresponding author; e-mail: magdalena.skurzok@uj.edu.pl instead of energy deposition measurement [8-16]. Therefore, it is crucial to perform precise time calibration of the detection setup.

The time calibration for the J-PET scanner is carried out based on measurements performed with a reference detector and radioactive sodium source. Collected data allowed us to perform a time calibration for each of the 192 scintillator strips (i.e. the time difference calibration of a single detection module), synchronize between the strips in a single cylindrical layer, as well as synchronize between the three scintillator layers. The time calibration method is briefly described in this report.

\section{J-PET calibration with a reference detector}

Measurements used for the calibration and synchronization of the J-PET detector modules was performed using a $5 \times 5 \times 19 \mathrm{~mm}^{3}$ BC-420 plastic reference detector coupled to a single photomultiplier and with a ${ }^{22} \mathrm{Na}$ source placed on it [17]. The whole system was mounted on a metal arm inside the J-PET detector as shown in Fig. 1. A single measurement is carried out with the reference detector pointing at the center of the scintillator strip to be measured, schematically presented in Fig. 2. The data for each detection module were taken in coincidence with signals from the reference detector which, due to small size of the reference scintillator, selects a well defined beam of gamma quanta annihilation for calibration. 


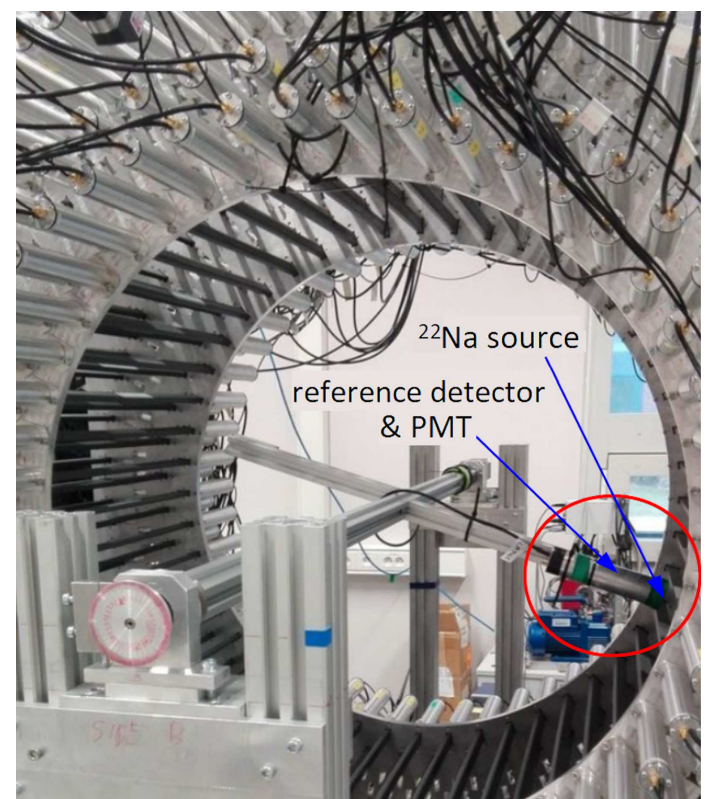

Fig. 1. A photo of the J-PET scanner with reference detector mounted inside the diagnostic chamber.

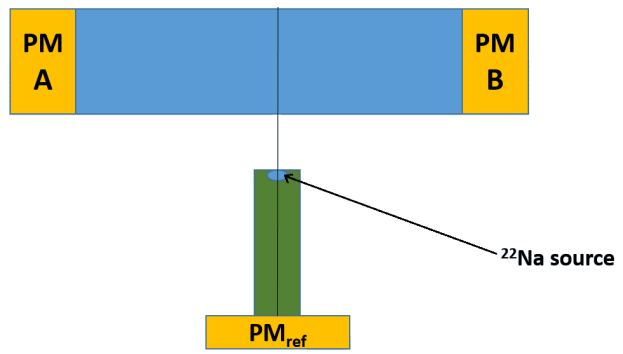

Fig. 2. Scheme of a measurement performed for a single J-PET detection module. The scintillator strip, reference detector, and photomultipliers are marked with blue, green, and yellow rectangles, respectively.

The measurement procedure was repeated for each of the 192 J-PET scintillator strips arranged in three cylindrical layers. Since the J-PET front-end electronics are able to probe signals at four different thresholds, and on both the leading and trailing edges $[18,19]$ the calibration was done for times measured on each threshold of both signal edges. Collected data were analysed using the J-PET Framework software [20] with respect to (wrt) the time calibration of each separate module (so called "A-B" synchronization) and wrt the TOF between scintillators.

The calibration was performed taking advantage of the fact that the beam of selected annihilation quanta hits each scintillator in the center. Thus, in an ideal case the difference between the times of the signals registered at both sides of a single scintillator $\Delta t_{A B}=t_{B}-t_{A}$ must be equal to zero. But in reality the measured times are shifted with respect to the true values by some constant factors accounting for the delays in the photomultipliers and electronic components. Thus, $t_{A}=t_{A}^{\text {true }}-$ of $f_{A}$ and $t_{B}=t_{B}^{\text {true }}-$ of $f_{B}$, and the time difference $\Delta t_{A B}$ will be non-zero. For the "A-B" synchronization $\left(c_{1}\right)$ we can determine the $\Delta t_{A B}$ distribution for each detection module. By performing a Gaussian fit to each of these distributions one can extract the effective time offsets $($ off $)$ :

$$
c_{1}=\text { off } f_{A}-\text { of } f_{B} \text {. }
$$

An example of a $\Delta t_{A B}$ spectrum for one of the scintillator strips in the first layer of the J-PET detector is presented in Fig. 3.

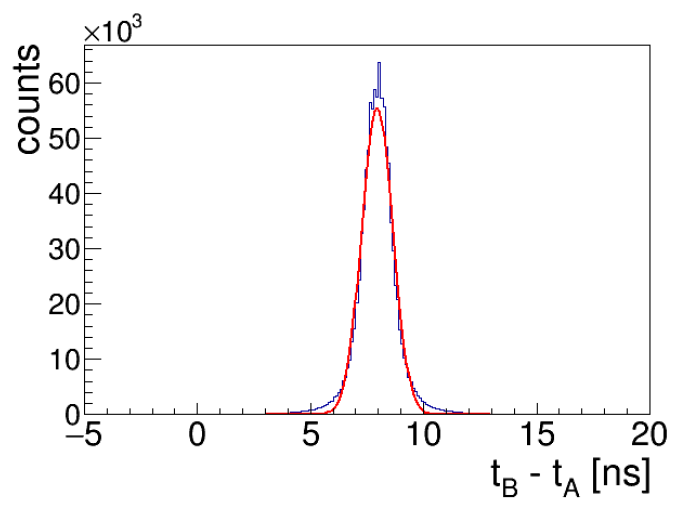

Fig. 3. Spectrum of the time difference between signals measured at two sides of a strip. Red curve presents Gaussian fit to the data.

In order to perform simultaneous synchronization of all modules in a single detector layer, a difference between the time of gamma quanta hit in the module and the time measured with the reference detector was determined $\Delta t_{r e f}=\left(t_{A}+t_{B}\right) / 2-t_{\text {ref }}$. Again, a fit to the $\Delta t_{\text {ref }}$ distributions gives the common reference time for all the modules, i.e. time synchronization, and gives the calibration constant $\left(c_{2}\right)$ related to the time offsets on both sides of a strip in the following way:

$$
c_{2}=-\left(\text { of } f_{B}+\text { of } f_{A}\right) / 2 \text {. }
$$

An example of a raw (without any selection conditions) $\Delta t_{\text {ref }}$ spectrum for a strip in the first J-PET layer is presented in Fig. 4.

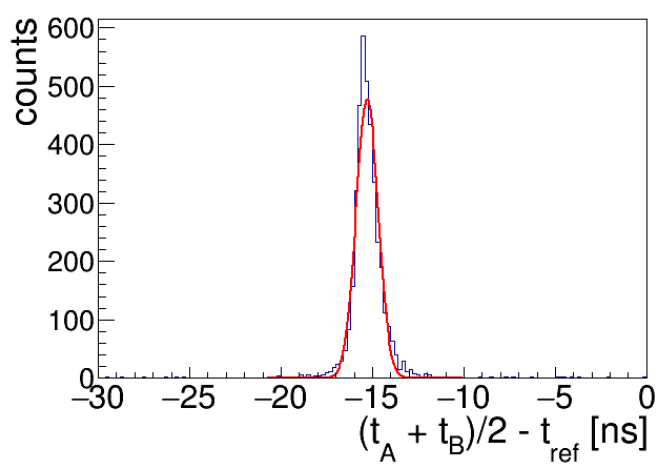

Fig. 4. Spectrum of time difference between qamma quanta hits in a J-PET module and reference detector $\Delta t_{r e f}=\left(t_{A}+t_{B}\right) / 2-t_{r e f}$. Red curve presents Gaussian fit to the data.

Solving the set of Eqs. (1) and (2) gives finally the following time offsets: 


$$
\begin{aligned}
& \text { of } f_{A}=c_{1} / 2-c_{2}, \\
& \text { of } f_{B}=-c_{1} / 2-c_{2} .
\end{aligned}
$$

Synchronization between layers was carried out wrt the first internal layer. The constant $c_{2}$ (in Eq. (3) and (4)) was then corrected for strips in the other layers $(L)$ with time constants $\Delta t_{L 2-L 1}$ and $\Delta t_{L 3-L 1}$ corresponding to the time elapsed for gamma quanta traveling from layer 1 to layer 2 , or to layer 3 , respectively. Time differences between layers were calculated based on known distances between layers and were found to be equal to $\Delta t_{L 2-L 1}=$ $0.1418 \pm 0.0033 \mathrm{~ns}$ and $\Delta t_{L 3-L 1}=0.5003 \pm 0.0033 \mathrm{~ns}$.

The time calibration method was validated with independent measurements performed using a collimated ${ }^{22} \mathrm{Na}$ radioactive source installed in the geometrical center of the J-PET barrel [21]. As an example, in Fig. 5 we show the $\Delta t_{A B}$ time difference for each strip of layer 2 before (upper part) and after applying calibration constants (lower part). As one can see, the $\Delta t_{A B}$ is distributed around zero as expected for a properly calibrated detector.

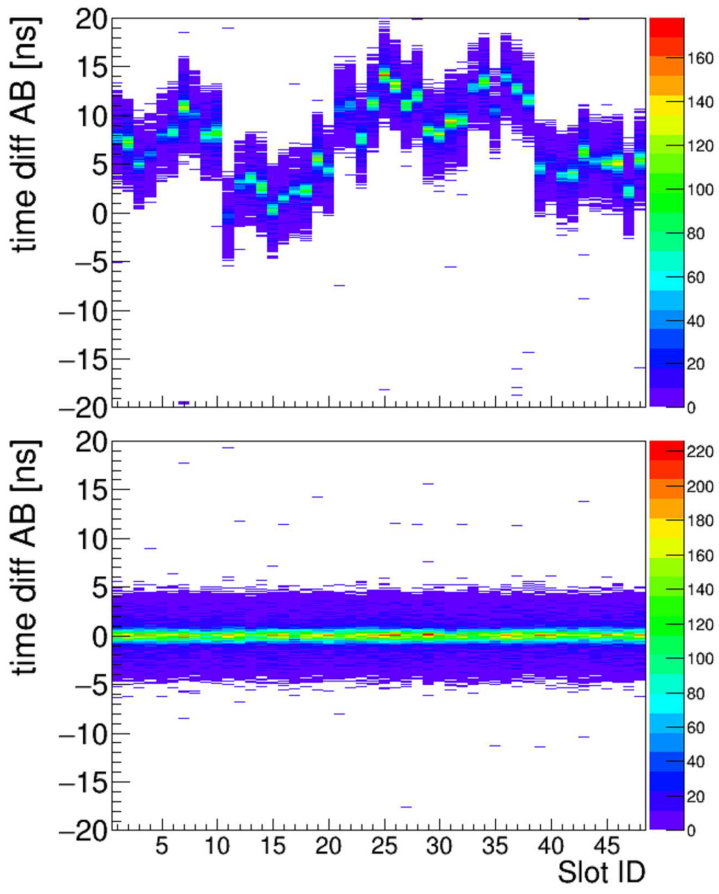

Fig. 5. Spectra of the $\Delta t_{A B}$ time difference as a function of the detection module number for the J-PET layer 2 obtained using collimated ${ }^{22} \mathrm{Na}$ source before (upper part) and after calibration (lower part).

The synchronization of modules can be checked by studying the TOF of annihilation gamma quanta for two modules located opposite to each other. TOF is defined as the difference between the measured times of two backto-back gamma quanta hits. Figure 6 shows the TOF spectrum for pairs of modules in layer 2 after the synchronization. As in the previous case we expect the distribution to be peaked around zero for all the modules since the source was placed in the geometrical center of the detector.

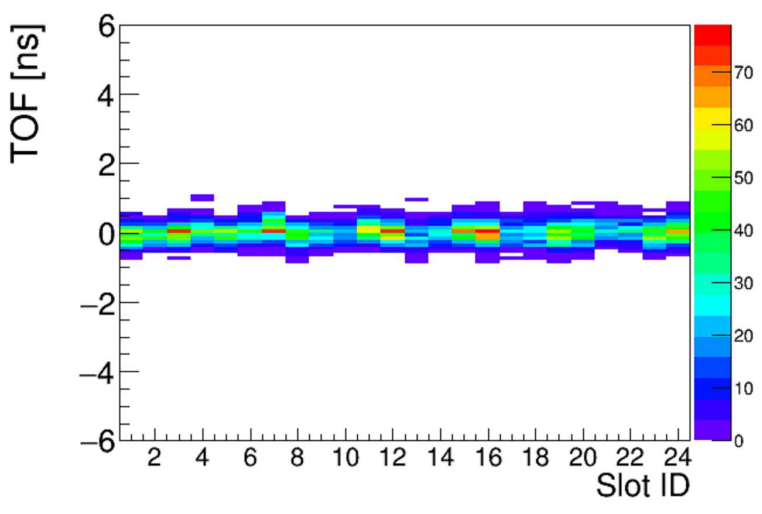

Fig. 6. Spectrum of time of flight (TOF) versus ID of the pair of opposite slots in the 2nd J-PET layer obtained for data taken with the collimated ${ }^{22} \mathrm{Na}$ source after the time calibration.

\section{Summary}

We have presented the method used for time calibration of the J-PET detector. It is based on data taken by irradiating each detector module with a radioactive sodium source in coincidence with reference detector. This data was used to calibrate the time difference measurement within each single module and for time synchronization of modules in all detector layers. The method was validated with independent measurements using a collimated ${ }^{22} \mathrm{Na}$ source placed in the center of the detector, demonstrating that the developed procedure gives satisfying results. There are other methods which may be used for the J-PET calibration and monitoring. For example, a measurement referenced to cosmic radiation [22]; the performance and limitations of this and other methods is now under investigation.

\section{Acknowledgments}

The authors acknowledge the technical support by A. Heczko, W. Migdał, the financial support from the Polish National Center for Development and Research through grant INNOTECH-K1/IN1/64/159174/NCBR/12, the EU and MSHE grant no. POIG.02.03.00-161 00-013/09, and the National Science Center Poland based on decision number DEC-2013/09/N/ST2/02180 and UMO2016/21/B/ST2/01222. B.C. Hiesmayr acknowledges gratefully the Austrian Science Fund FWF-P26783.

\section{References}

[1] P.J. Slomka, T. Pan, G. Germano, Semin. Nucl. Med. 46, 5 (2016).

[2] S. Vandenberghe, E. Mikhaylova, E. D'Hoe, P. Mollet, J.S. Karp, EJNMMI Phys. 3, 3 (2016).

[3] J.S. Karp, S. Surti, M.E. Daube-Witherspoon, G. Muehllehner, J. Nucl. Med. 49, 462 (2008).

[4] J.J. Griesmer, T.L. Laurence, Patent No. US7414246, 2008.

[5] T. Laurence, J.J. Griesmer, Patent Application No. US78209075, 2010.

[6] G. Muehllehner, J.S. Karp, Patent No. US7557350, 2009. 
[7] C.W. Stearns, Patent No. US5272343, 1993.

[8] P. Moskal, Sz. Niedźwiecki, T. Bednarski, E. Czerwiński, Ł. Kapłon, E. Kubicz, I. Moskal, M. PawlikNiedźwiecka, N.G. Sharma, M. Silarski, M. Zieliński, N. Zoń, P. Białas, A. Gajos, A. Kochanowski, G. Korcyl, J. Kowal, P. Kowalski, T. Kozik, W. Krzemień, M. Molenda, M. Pałka, L. Raczyński, Z. Rudy, P. Salabura, A. Słomski, J. Smyrski, A. Strzelecki, A. Wieczorek, W. Wiślicki, Nucl. Instrum. Methods Phys. Res. A 764, 317 (2014).

[9] A. Gajos, D. Kamińska, E. Czerwiński, D. Alfs, T. Bednarski, P. Białas, B. Głowacz, M. Gorgol, B. Jasińska, Ł. Kapłon, G. Korcyl, P. Kowalski, T. Kozik, W. Krzemień, E. Kubicz, M. Mohammed, Sz. Niedźwiecki, M. Pałka, M. Pawlik-Niedźwiecka, L. Raczyński, Z. Rudy, O. Rundel, N.G. Sharma, M. Silarski, A. Słomski, A. Strzelecki, A. Wieczorek, W. Wiślicki, B. Zgardzińska, M. Zieliński, P. Moskal, Nucl. Instrum. Methods Phys. Res. A 819, 54 (2016).

[10] D. Kamińska, A. Gajos, E. Czerwiński, D. Alfs, T. Bednarski, P. Białas, C. Curceanu, K. Dulski, B. Głowacz, N. Gupta-Sharma, M. Gorgol, B.C. Hiesmayr, B. Jasińska, G. Korcyl, P. Kowalski, W. Krzemień, N. Krawczyk, E. Kubicz, M. Mohammed, Sz. Niedźwiecki, M. Pawlik-Niedźwiecka, L. Raczyński, Z. Rudy, M. Silarski, A. Wieczorek, W. Wiślicki, B. Zgardzińska, M. Zieliński, P. Moskal, Eur. Phys. J. C 76, 445 (2016).

[11] P. Moskal, D. Alfs, T. Bednarski, P. Białas, E. Czerwiński, C. Curceanu, A. Gajos, B. Głowacz, M. Gorgol, B.C. Hiesmayr, B. Jasińska, D. Kamińska, G. Korcyl, P. Kowalski, T. Kozik, W. Krzemień, N. Krawczyk, E. Kubicz, M. Mohammed, Sz. Niedźwiecki, M. Pawlik-Niedźwiecka, L. Raczyński, Z. Rudy, M. Silarski, A. Wieczorek, W. Wiślicki, M. Zieliński, Acta Phys. Pol. B 47, 509 (2016).

[12] P. Moskal, O. Rundel, D. Alfs, T. Bednarski, P. Białas, E. Czerwiński, A. Gajos, K. Giergiel, M. Gorgol, B. Jasińska, D. Kamińska, Ł. Kapłon, G. Korcyl, P. Kowalski, T. Kozik, W. Krzemień, E. Kubicz, Sz. Niedźwiecki, M. Pałka, L. Raczyński, Z. Rudy, N.G. Sharma, A. Słomski, M. Silarski, A. Strzelecki, A. Wieczorek, W. Wiślicki, P. Witkowski, M. Zieliński, N. Zoń, Phys. Med. Biol. 61, 2025 (2016).

[13] P. Moskal, N. Zoń, T. Bednarski, P. Białas, E. Czerwiński, A. Gajos, D. Kamińska, Ł. Kapłon, A. Kochanowski, G. Korcyl, J. Kowal, P. Kowalski, T. Kozik, W. Krzemień, E. Kubicz, Sz. Niedźwiecki, M. Pałka, L. Raczyński, Z. Rudy, O. Rundel, P. Salabura, N.G. Sharma, M. Silarski, A. Słomski, J. Smyrski, A. Strzelecki, A. Wieczorek, W. Wiślicki, M. Zieliński, Nucl. Instrum. Methods Phys. Res. A 775, 54 (2015).

[14] L. Raczynski, P. Moskal, P. Kowalski, W. Wiślicki, T. Bednarski, P. Białas, E. Czerwiński, Ł. Kapłon, A. Kochanowski, G. Korcyl, J. Kowal, T. Kozik, W. Krzemień, E. Kubicz, M. Molenda, Sz. Niedźwiecki, M. Pałka, M. Pawlik, Z. Rudy, P. Salabura, N.G. Sharma, M. Silarski, A. Słomski J. Smyrski, A. Strzelecki, A. Wieczorek, M. Zieliński, N. Zoń, Nucl. Instrum. Methods Phys. Res. A 764, 186 (2014).
[15] L. Raczynski, P. Moskal, P. Kowalski, W. Wiślicki, T. Bednarski, P. Białas, E. Czerwiński, A. Gajos, Ł. Kapłon, A. Kochanowski, G. Korcyl, J. Kowal, T. Kozik, W. Krzemień, E. Kubicz, Sz. Niedźwiecki, M. Pałka, Z. Rudy, O. Rundel, P. Salabura, N.G. Sharma, M. Silarski, A. Słomski, J. Smyrski, A. Strzelecki, A. Wieczorek, M. Zieliński, N. Zoń, Nucl. Instrum. Methods Phys. Res. A 786, 105 (2015).

[16] L. Raczynski, W. Wiślicki, W. Krzemień, P. Kowalski, D. Alfs, T. Bednarski, P. Białas, C. Curceanu, E. Czerwiński, K. Dulski, A. Gajos, B. Głowacz, M. Gorgol, B. Hiesmayr, B. Jasińska, D. Kamińska, G. Korcyl, T. Kozik, N. Krawczyk, E. Kubicz, M. Mohammed, M. Pawlik-Niedźwiecka, S. Niedźwiecki, M. Pałka, Z. Rudy, O. Rundel, N. Gupta-Sharma, M. Silarski, J. Smyrski, A. Strzelecki, A. Wieczorek, B. Zgardzińska, M. Zieliński, P. Moskal, Phys. Med. Biol. 62, 5076 (2017).

[17] T. Bednarski, Ph.D. Thesis, 2016.

[18] G. Korcyl, D. Alfs, T. Bednarski, P. Białas, E. Czerwiński, K. Dulski, A. Gajos, B. Głowacz, B. Jasińska, D. Kamińska, Ł. Kapłon, P. Kowalski, T. Kozik, W. Krzemień, E. Kubicz, M. Mohammed, Sz. Niedźwiecki, M. Pałka, M. Pawlik-Niedźwiecka, L. Raczyński, Z. Rudy, O. Rundel, N.G. Sharma, M. Silarski, A. Słomski, K. Stoła, A. Strzelecki, A. Wieczorek, W. Wiślicki, B.K. Zgardzińska, M. Zieliński, P. Moskal, Acta Phys. Pol. B 47, 491 (2016).

[19] M. Pałka, P. Strzempek, G. Korcyl, T. Bednarski, Sz. Niedźwiecki, P. Białas, E. Czerwiński, K. Dulski, A. Gajos, B. Głowacz, M. Gorgol, B. Jasińska, D. Kamińska, M. Kajetanowicz, P. Kowalski, T. Kozik, W. Krzemień, E. Kubicz, M. Mohammed, L. Raczyński, Z. Rudy, O. Rundel, P. Salabura, N.G. Sharma, M. Silarski, J. Smyrski, A. Strzelecki, A. Wieczorek, W. Wiślicki, M. Zieliński, B. Zgardzińska, P. Moskal, JINST 12, P08001 (2017).

[20] W. Krzemień, A. Gajos, A. Gruntowski, K. Stola, D. Trybek, T. Bednarski, P. Białas, E. Czerwiński, D. Kamińska, L. Kapłon, A. Kochanowski, G. Korcyl, J. Kowal, P. Kowalski, T. Kozik, E. Kubicz, P. Moskal, Sz. Niedźwiecki, M. Pałka, L. Raczyński, Z. Rudy, P. Salabura, N.G. Sharma, M. Silarski, A. Słomski, J. Smyrski, A. Strzelecki, A. Wieczorek, W. Wiślicki, M. Zieliński, N. Zoń, et al., Acta Phys. Pol. A 127, 1491 (2015).

[21] E. Kubicz, M. Silarski, A. Wieczorek, D. Alfs, T. Bednarski, P. Białas, E. Czerwiński, A. Gajos, B. Głowacz, B. Jasińska, D. Kamińska, G. Korcyl, P. Kowalski, T. Kozik, W. Krzemień, M. Mohammed, I. Moskal, S. Niedźwiecki, M. Pawlik-Niedźwiecka, L. Raczyński, Z. Rudy, A. Strzelecki, W. Wiślicki, M. Zieliński, B. Zgardzińska, P. Moskal, Acta Phys. Pol. B 47, 537 (2016).

[22] M. Silarski, E. Czerwiński, T. Bednarski, P. Moskal, P. Białas, Ł. Kapłon, A. Kochanowski, G. Korcyl, J. Kowal, P. Kowalski, T. Kozik, W. Krzemień, M. Molenda, Sz. Niedźwiecki, M. Pałka, M. Pawlik, L. Raczyński, Z. Rudy, P. Salabura, N.G. Sharma, A. Słomski, J. Smyrski, A. Strzelecki, W. Wiślicki, M. Zieliński, N. Zoń, Bio-Alg. Med.-Syst. 10, 19 (2014). 